\title{
Monitoring peroxide value in oxidized emulsions by Fourier transform infrared spectroscopy
}

\begin{abstract}
Soybean oil (SBO) was blended with 10-40\% palm kernel olein (PKO) to obtain SBO:PKO blends with different degrees of unsaturation. Oil-in-water $(\mathrm{O} / \mathrm{W})$ emulsions were then prepared with 70 wt-\% of SBO or SBO:PKO blends and monitored for their chemical destabilization after an accelerated oxidation process up to 12 days at $60^{\circ} \mathrm{C}$. The formation rate of hydroperoxides, as demonstrated by peroxide value (PV) evolution, throughout the oxidation period was relatively high for a fully SBO-based emulsion as compared to those with PKO incorporation. Fourier transform infrared (FTIR) spectroscopic method was also performed in parallel with PV determination, providing further information on structural changes of the functional groups due to lipid oxidation in the emulsions. By using a partial least square chemometric method, a developed calibration model that was based on the spectral region between 1800 and $1480 \mathrm{~cm}-1$ was shown to be able to predict the PV in oxidized emulsions over the range of $4-45 \mathrm{meq} / \mathrm{kg}$. With a 'leave-one-out' cross-validation optimization procedure, the calibration model provides a good coefficient of determination of 0.98 and a root mean standard error of cross-validation of 2.09. In general, FTIR spectroscopy is a suitable technique to monitor PV in oxidized emulsions. (C) 2005 WileyVCH Verlag GmbH \& Co. KGaA.
\end{abstract}

Keyword: Emulsion destabilization; FTIR; Lipid oxidation; Palm kernel olein; Peroxide value 